\title{
A Rapid Lysostaphin Production Approach and a Convenient Novel Lysostaphin Loaded Nano-emulgel; As a Sustainable Low-Cost Methicillin-Resistant Staphylococcus aureus Combating Platform
}

\author{
Hanzada T. Nour El-Din ${ }^{1}$, Noha M. Elhosseiny ${ }^{1}\left(\mathbb{D}\right.$, Mohamed A. El-Gendy ${ }^{2}$, \\ Azza A. Mahmoud ${ }^{2}$ (D), Manal M. M. Hussein ${ }^{1,3}$ and Ahmed S. Attia 1,*(D) \\ 1 Department of Microbiology and Immunology, Faculty of Pharmacy, Cairo University, Cairo 11562, Egypt; \\ Hanzada.noreldin@pharma.cu.edu.eg (H.T.N.E.-D.); noha.elhuseiny@pharma.cu.edu.eg (N.M.E.); \\ manal.maher@pharma.cu.edu.eg (M.M.M.H.) \\ 2 Department of Pharmaceutics and Pharmaceutical Technology, Faculty of Pharmaceutical Sciences and \\ Pharmaceutical Industries, Future University in Egypt, Cairo 11835, Egypt; \\ Mohamed.Emad@fue.edu.eg (M.A.E.-G.); azza.ahmed@fue.edu.eg (A.A.M.) \\ 3 Department of Microbiology \& Immunology, School of Pharmacy, New Giza University, Giza 12256, Egypt \\ * Correspondence: ahmed.attia@pharma.cu.edu.eg; Tel.: +20-010-65344060; Fax: +20-2-23628246
}

Received: 19 February 2020; Accepted: 10 March 2020; Published: 12 March 2020

\begin{abstract}
Staphylococcus aureus is a Gram-positive pathogen that is capable of infecting almost every organ in the human body. Alarmingly, the rapid emergence of methicillin-resistant $S$. aureus strains (MRSA) jeopardizes the available treatment options. Herein, we propose sustainable, low-cost production of recombinant lysostaphin (rLST), which is a native bacteriocin destroying the staphylococcal cell wall through its endopeptidase activity. We combined the use of E. coli BL21(DE3)/pET15b, factorial design, and simple Ni-NTA affinity chromatography to optimize rLST production. The enzyme yield was up to $50 \mathrm{mg} / \mathrm{L}$ culture, surpassing reported systems. Our rLST demonstrated superlative biofilm combating ability by inhibiting staphylococcal biofilms formation and detachment of already formed biofilms, compared to vancomycin and linezolid. Furthermore, we aimed at developing a novel rLST topical formula targeting staphylococcal skin infections. The phase inversion composition (PIC) method fulfilled this aim with its simple preparatory steps and affordable components. LST nano-emulgel (LNEG) was able to extend active LST release up to $8 \mathrm{~h}$ and cure skin infections in a murine skin model. We are introducing a rapid, convenient rLST production platform with an outcome of pure, active rLST incorporated into an effective LNEG formula with scaling-up potential to satisfy the needs of both research and therapeutic purposes.
\end{abstract}

Keywords: lysostaphin; sustainable; factorial-design; optimization; low-cost; nano-emulgel; skin infections

\section{Introduction}

Since their clinical introduction in the 1930s, antibiotics have highly impacted human morbidity and mortality. Unfortunately, decades of antibiotics mis-use has applied selective pressure on pathogens, which results in unprecedented antimicrobial resistance [1,2]. S. aureus is a leading cause of hospital and community infections worldwide [3]. However, the most recent antimicrobial for combating staphylococcal infections was discovered 30 years ago. To fill this gap in a way that considerably lower the resistance potential, new non-conventional ways had to be explored. Investigated alternatives include plant-derived compounds [4], bacteriophages and phage lysins [5], RNA-based therapeutics [6], antimicrobial adjuvants [7], and antimicrobial peptides (AMP) [8]. 
Bacteriocins, which are one subgroup of AMPs, are ribosomal-synthesized proteinaceous bactericidal compounds produced by immune bacteria including one of which is lysostaphin (LST). LST, which is also termed as a bacteriolysin, is an endopeptidase discovered in the 1960s as an anti-staphylococcal agent destroying the bacterial cell wall, which makes it a promising therapeutic alternative to treat staphylococcal infections, starting from skin and moving to systemic infections $[9,10]$. The pharmaceutical industry has been revolutionized by a variety of formulas, among which are the nano-emulsions (NEs). NE is defined as a disperse system with droplets of less than $100 \mathrm{~nm}$ [11]. This small size not only imparts advantageous physical properties, such as optical clarity and elastic behavior, but also makes NEs stable against sedimentation and creaming [12]. Moreover, NEs are thermodynamically stable, and can be used when aiming at controlled drug release. Topically, NEs, specifically the water in oil ones, has better adhesion on the skin surface, which leads to a larger concentration gradient and, hence, better targeting for skin infections. In addition, topical NEs are non-greasy, easily spreadable, and removable when enhancing patients' compliance [13]. Commercially, NEs are gaining more focus due to their simple preparatory procedures and the affordability of the materials in use. This reduces their overall production cost [14] and, as a result, they are already used as vehicles for drugs active against herpes labialis, topical fungal, and bacterial infections and vaginitis [15].

In the present study, we aim to optimize recombinant lysostaphin (rLST) production and treat staphylococcal skin infections topically under the umbrella of swiftness, affordability, and simplicity. This could enable countries with minimal resources to make full use of lysostaphin applications with the potential for scale up production.

\section{Materials and Methods}

\subsection{Statement of Ethical Approval}

All animal procedures were approved by the Research Ethics Committee of the Faculty of Pharmacy, Cairo University (approval\# MI 1613), following the Guide for the Care and Use of Laboratory Animals published by the Institute of Laboratory Animal Research (Washington, DC, USA).

\subsection{Bacterial Strains and Culture Conditions}

Staphylococcal strains used in this study included: S. aureus strains Newman [16] and USA300, which is a multiple antibiotic resistant and community-acquired strain [17], the S. simulans strain TNK3 [18], the S. epidermidis strain ATCC 12228 (ATCC), and the S. lugdunensis strain N920143 [19]. Escherichia coli strain TOP10 (Invitrogen) was used for cloning and strain BL21 (DE3) [20] used for the rLST production. Staphylococcal strains were grown aerobically at $37^{\circ} \mathrm{C}$ in tryptic soy broth (TSB). For E. coli strains, they were grown aerobically at $37^{\circ} \mathrm{C}$ in Luria Bertani (LB) broth. When appropriate, LB was supplemented with ampicillin at a final concentration of $100 \mu \mathrm{g} / \mathrm{mL}$.

\subsection{Cloning and Expression of Recombinant Lysostaphin in E. coli}

Primer pair AA662 (5'-GGGcatatgGCTGCAACACATGAACA-3', NdeI site underlined) and AA663 (5'-GGGctcgagTTACTTTATAGTTCCCCAAAG-3', XhoI site underlined and stop codon in italics) was used to amplify a $738 \mathrm{bp}$ fragment encoding mature lysostaphin [21], using purified chromosomal S. simulans DNA as a template. The PCR product was column purified, and then double digested using NdeI and XhoI (NEB). The digested PCR product was then ligated into plasmid pET15b (Novagen, Madison, MA, USA) and transformed into the E. coli TOP10 cells. The resulting plasmid was verified by DNA sequencing and designated pET15b-lyso. Then it was transformed into E. coli BL21 (DE3) for protein production. To screen for successful lysostaphin production, a BL21/ pET15b-lyso overnight culture was used to inoculate fresh LB medium in a ratio of $1: 100$, and grown at $37^{\circ} \mathrm{C}$ to mid-log phase, which is followed by induction with $0.5 \mathrm{mM}$ isopropyl-D-1-thiogalactopyranoside (IPTG) for $3 \mathrm{~h}$ at $30^{\circ} \mathrm{C}$. The cells were collected by centrifugation at $4000 \times g$ at $4{ }^{\circ} \mathrm{C}$, and disrupted 
by sonication at $40 \%$ amplitude, and 3-s pulses for $10 \mathrm{~min}$. The lysate was centrifuged and the clear supernatant was purified with Ni-NTA resin (Qiagen, Hilden, Germany) as described earlier [22] and, then, analyzed on a $12.5 \%$ sodium dodecyl sulphate polyacrylamide gel electrophoresis (SDS-PAGE). When needed, the recombinant lysostaphin (rLST) elution buffer was exchanged with $0.1 \mathrm{M}$ phosphate buffer pH 7.5 using the Zeba ${ }^{\mathrm{TM}}$ Spin Desalting Columns, 7K MWCO (Thermo Fisher, Waltham, WA, USA).

\subsection{Optimization of Recombinant Lysostaphin Production}

Full factorial design using the statistical software package Minitab 16 (Minitab Inc., State College, Pennsylvania, PA, USA) was applied to evaluate the influence of five independent factors (temperature, IPTG-concentration, growth medium type, induction time, and agitation speed) against the dependent variable of the rLST yield in $\mathrm{mg} / \mathrm{L}$ culture. The low $(-)$, basal (0), and high $(+)$ levels for the studied factors were as follows, respectively: temperature $\left(16,30\right.$, and $\left.37^{\circ} \mathrm{C}\right)$, IPTG concentration $(0.25,0.5$, and $1 \mathrm{mM}$ ), type of growth medium (nutrient broth (NB), LB, and TSB), induction time ( 3,6 , and $18 \mathrm{~h}$ ), and agitation speed (100, 180, and $250 \mathrm{rpm})$. Following each run, the purified lysostaphin was quantitated using a nanophotometeric device (Implen, California, CA, USA) and the QuantiPro BCA assay kit (Sigma-Aldrich, St. Louis, Missouri, MI, USA). In addition, aliquots of each elution fraction were analyzed by SDS-PAGE.

\subsection{Analyses of the Different Factors and Their Interactions on the Production of rLST}

The standardized effect of each factor (E-value) was calculated using Minitab 16. The E-value magnitude of the tested factor indicated its effect or its significance in affecting the response, while its sign indicated its positive or negative influence on the responses. The interacting factors were determined by presenting the analysis of variance (ANOVA). The main and interaction effects of each factor having $p$ values $\leq 0.05$ were considered as potentially significant.

To investigate the interplay of the evaluated factors, interaction plots, expressed in terms of rLST conc. $\mathrm{mg} / \mathrm{L}$, were constructed. Parallel lines indicate the absence of an interaction, whereas non-parallel lines indicate the presence of an interaction. The F-value of this test determines whether a group of terms is associated with the response. A sufficiently large F-value indicates statistical significance.

\subsection{Lysostaphin Activity Assay}

The bacteriolytic activity of rLST was determined as described before [23]. Overnight cultures of the staphylococcal strains mentioned above $(18 \mathrm{~h})$ were pelleted and the cells were then resuspended in a $0.1 \mathrm{M}$ phosphate buffer $\mathrm{pH} 7.5$ to $\mathrm{OD}_{600}$ of 0.25 . The cell suspension was preincubated at $37^{\circ} \mathrm{C}$ for $10 \mathrm{~min}$, and then $50 \mu \mathrm{L}$ of rLST preparation was added. One unit (U) of activity is defined as the amount of preparation causing $50 \%$ reduction in the turbidity of a $6-\mathrm{mL}$ cell suspension within $10 \mathrm{~min}$ at $37^{\circ} \mathrm{C}$ in a $10-\mathrm{mm}$ cuvette.

\subsection{Biofilms Combating Assays}

\subsubsection{Determination of the Minimum Inhibitory Concentration (MIC)}

MIC of rLST, vancomycin, and linezolid were determined for all the five tested staphylococcal strains using the two-fold dilution method according to the Clinical and Laboratory Standards Institute (CLSI) guidelines [24].

\subsubsection{Biofilm Formation Inhibition Assay (Pre-Exposure)}

Staphylococcal cells were gown for $18 \mathrm{~h}$ in TSB and then cultures were normalized to $\mathrm{OD}_{600}$ of 1 and diluted 1:100 in fresh TSB. Diluted cultures $(100 \mu \mathrm{L})$ were mixed with equal volume of either rLST (20 U), or a solution containing the MIC value of vancomycin or linezolid to have a final concentration of half the MIC (for the respective strain) and placed in the wells of an untreated polystyrene, 96-well 
flat-bottomed plate. The plates were incubated statically at $37^{\circ} \mathrm{C}$ for $24 \mathrm{~h}$. Wells were washed three times with phosphate buffered saline (PBS). The washing step was done by gently submerging the plate in a small tub of PBS, and then shaking the water off and, lastly, blot dry on a stack of paper towels and leave to dry overnight [25]. Adherent cells were stained with crystal violet $(0.4 \% w / v)$ at room temperature for $15 \mathrm{~min}$. Then wells were washed, $150 \mu \mathrm{L}$ of absolute ethanol were added, and absorbance at $\mathrm{OD}_{595}$ was recorded. The biofilm formation ability was evaluated using a biofilm formation index [BFI]: (ODCV Biofilm - ODCV Control)/ODPlanktonic [26,27].

\subsubsection{Biofilm Detachment Assay (Post-Exposure)}

An $18 \mathrm{~h}$ TSB cultures of staphylococci were normalized to $\mathrm{OD}_{600}$ of 1 , and then diluted 1:100 with fresh TSB. Individual wells of a 96-well flat-bottomed plates were filled with $0.1 \mathrm{~mL}$ aliquots of the diluted culture. The plates were incubated for $24 \mathrm{~h}$ at $37^{\circ} \mathrm{C}$. Then the $\mathrm{OD}_{600}$ of the wells was measured after the incubation for normalization purposes. Growth medium and the unattached cells were discarded, and the biofilms were washed three times with PBS. A washing step was done by gently submerging the plate in a small tub of PBS, then shaking the buffer off, and, lastly, blot dry the plate on a stack of paper towels and leave to dry overnight. Next, $50 \mu \mathrm{L}$ of either rLST (20 U), vancomycin $(400 \mu \mathrm{g} / \mathrm{mL})$, or linezolid $(800 \mu \mathrm{g} / \mathrm{mL})$ were mixed with $50 \mu \mathrm{L}$ of TSB/well and added to the plates. Untreated biofilms (100\% reference values) were obtained by adding $50 \mu \mathrm{L}$ of ultrapure water instead of the antimicrobial agents. The plates were incubated at $37^{\circ} \mathrm{C}$ for $24 \mathrm{~h}$ [28]. The rest of the protocol was done as described above.

\subsection{Incorporation of the rLST into Nano-Emulgel Preparation}

The nano-emulgel was prepared using the phase inversion composition method (PIC) [29]. The vehicle was prepared by mixing $0.2 \mathrm{~g}$ isopropyl myristate as the oil phase, $0.7 \mathrm{~g}$ Tween 80 as a surfactant, and $0.1 \mathrm{~g}$ polyethylene glycol 400 as a co-surfactant by vortexing for $60 \mathrm{~s}$. Then, a $0.67 \mathrm{~g}$ aqueous phase containing $1 \mathrm{mg}$ rLST were added stepwise with continuous mixing.

\subsubsection{Characterization of the rLST Nano-Emulgel}

Particle size and morphological examination of the prepared formula were done using a high resolution-transmission electron microscope (HR-TEM - JEOL2100, JEOL Ltd., Akishima, Japan) coupled with a Gatan axis-mount 2kx2k digital camera (Gatan, Inc., California, CA, USA).

\subsubsection{In-Vitro Release Study}

The experiment was conducted in a vertical Franz diffusion cell apparatus with an acceptor compartment volume of $6.8 \mathrm{~mL}$, which has a membrane filter (HT-450 Tuffryn membrane with $0.45 \mu \mathrm{m}$ pore size, Pall Life Science, New York, NY, USA) and diffusion area of $1.767 \mathrm{~cm}^{2}$ (MicroettePlus ${ }^{\mathrm{TM}}$, Hanson Research, California, CA, USA). For the donor compartment, an amount of $0.25-0.3 \mathrm{~g}$ of the formulation was initially set on the cellulose membrane and the appearance of the lysostaphin protein was monitored in the receptor compartment, containing PBS, pH 7.4) at 0, 0.5, 1, 2, 3, 4, 5, 6, 7, 8, 12 , and $24 \mathrm{~h}$. The withdrawn samples were then measured for the rLST protein content and the activity of the released rLST was determined as described above.

\subsection{Testing the Efficacy of the rLST Nano-Emulgel in an In-Vivo Murine Skin Model of Infection}

An in vivo murine methicillin-resistant $S$. aureus strains (MRSA) skin infection study was conducted as described before [30,31]. One day prior to the experiment, the backs of the six-eight-week-old BALB/C mice (Theodor Bilhariz Research Institute, Giza, Egypt) were shaved. Three mice groups $(n=5)$ received an intradermal injection $(40 \mu \mathrm{L})$ of MRSA USA300 containing $3 \times 10^{7} \mathrm{CFU}$. Forty-eight hours after infection, an open wound/abscess was formed at the site of injection. The first group was treated topically with the rLST nano-emulgel. The rLST nano-emulgel was prepared using the PIC 
method and it was applied three times daily using a disposable sterile cotton swab for each mouse at every application event. The second group was treated with the vehicle alone, while the control group did not receive any treatment. The respective mice were treated three times daily for five days. Twenty-four hours after the last treatment, mice were humanely euthanized using an over-dose of anesthesia followed by cervical dislocation. The wound area $\left(\sim 1 \mathrm{~cm}^{2}\right)$ was excised and homogenized in $0.5 \mathrm{~mL}$ of PBS. The homogenized skins were serially diluted and, then, aliquots of each dilution were plated on Mannitol Salt Agar (MSA) plates for viable counting. The extent of the lesion formation and ulceration was recorded using a digital camera.

\section{Results}

\subsection{Cloning and Recombinant Lysostaphin Production}

The DNA fragment encoding the mature lysostaphin estimated to be 738 bp was successfully amplified (Figure 1A). Then it was cloned in the expression vector pET15b and expressed. The His-tagged fragment was predicted to be approximately $29 \mathrm{kDa}$ (Figure 1B) based on the prediction using the ProtParam tool (https://web.expasy.org/protparam/) [32]. A band was visualized on an SDS-PAGE gel corresponding to the predicted size of the produced fragment (Figure 1C).

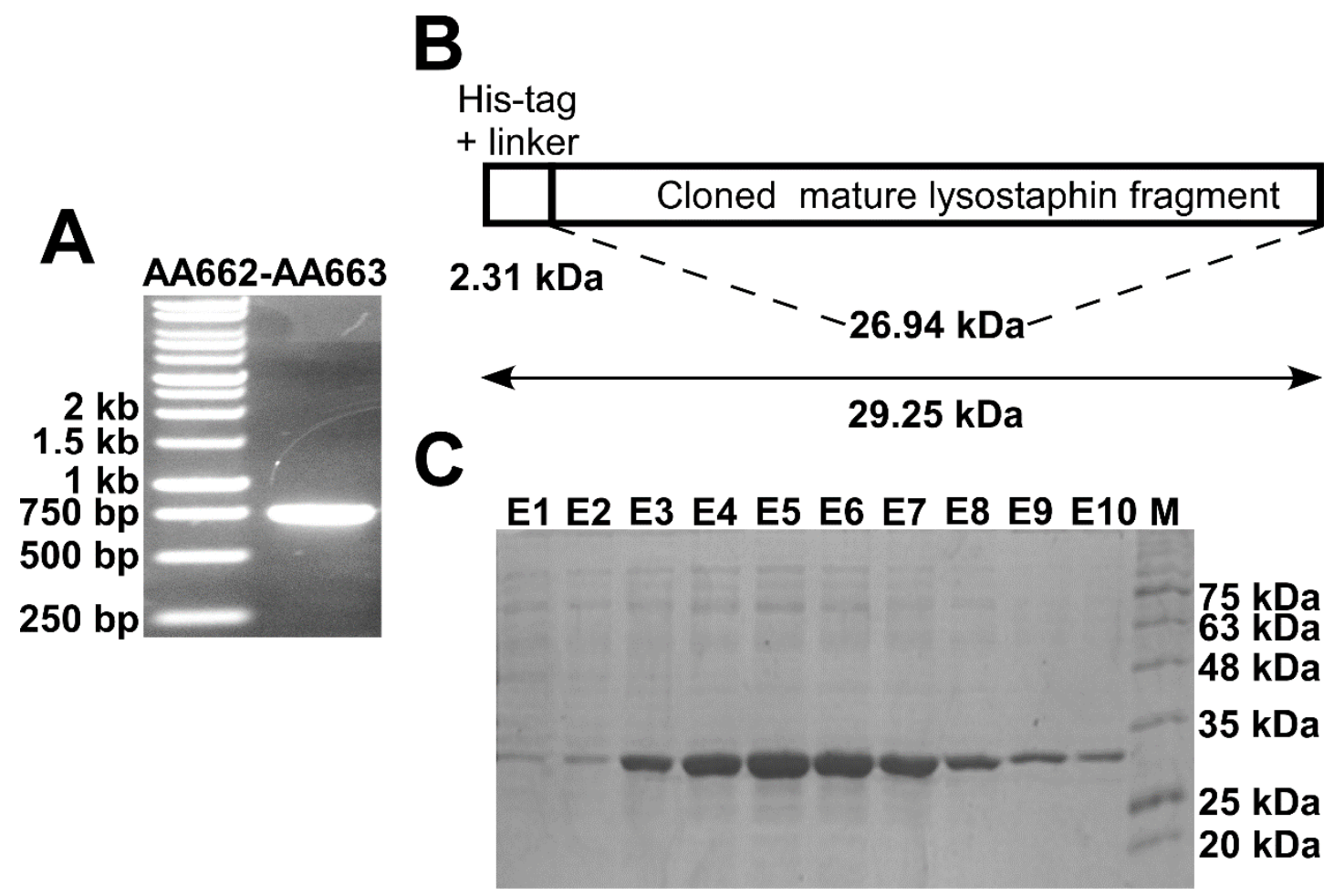

Figure 1. Cloning, production, and purification of rLST in E. coli. (A). Photograph of a 1\% agarose gel showing the PCR product of $\sim 740$ bp produced by the reaction using the primer pair AA662-AA663. The first lane of the gel contained a DNA ladder for size estimation. (B). Schematic diagram showing the expected size of the encoded lysostaphin His-tagged protein fragment based on the ProtParam tool. (C). Photograph of a 12.5\% SDS-PAGE gel stained with Coomassie Brilliant Blue showing elution fractions (E1-10) following Ni-NTA agarose beads purification. A prominent band representing the purified protein appeared $\sim 29 \mathrm{kDa}$. The last lane contained a protein molecular weight marker for the size estimation.

\subsection{Optimization of rLST Production}

The main effects analyses indicated that changing the growth media from NB to the much richer medium TSB had the greatest positive effect $(\mathrm{E}-\mathrm{value}=13.362, p<0.001)$. On the other hand, increasing 
the IPTG concentration from 0.25 to $1 \mathrm{mM}$ was the only factor that had a negative impact on the yield of the rLST (E-value $=-1.137)$. However, it was not statistically significant $(p>0.05)$. Regarding temperature, induction time, and agitation speed, there were very slight non-statistically significant positive effects $(p>0.05)$ for shifting to the high level of these three variables $\left(37^{\circ} \mathrm{C}, 18 \mathrm{~h}\right.$, and $250 \mathrm{rpm}$, respectively) on the rLST yield (E-value $=1.101,1.500$, and 1.636, respectively). The rLST yield varied from $1.2 \pm 0.57 \mathrm{mg} / \mathrm{L}$ to $50.5 \pm 0.99 \mathrm{mg} / \mathrm{L}$ culture (Table S1).

The strength of the model was tested by the normal probability plot of residuals (Figure 2A). The straight line in the graph represented the expected data, while the dots represented the actual observed data. A coefficient of $(\mathrm{R}=0.95)$ suggested the fitted model could explain $95 \%$ of the total variation. The standardized effects' magnitude and significance of each factor on its own or in combination with other factors are represented in the form of a Pareto chart (Figure 2B). Thirteen factors or factors' interactions showed significant effects on the yield of the rLST, as indicated by the extension of their standardized effect beyond the reference line. Among those 13 factors/factors interactions, the growth media factor was the only standardized factor by itself that had a significant positive effect $(E=13.362)$. Moreover, it was the one with the highest positive significant effect. On the other hand, seven factor interactions had a significant negative effect with the interaction between the temperature and the induction time having the highest significant negative effect $(E=-14.302)$. In addition, the interaction between the temperature, IPTG concentration, and the agitation speed had the least significant negative effect $(E=-2.719)$. Lastly, the interaction between the temperature and the growth media had the lowest positive significant effect $(E=3.035)$.

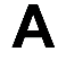

Normal Probability Plot ( Response is yield $\mathrm{mg} / \mathrm{L}$ )

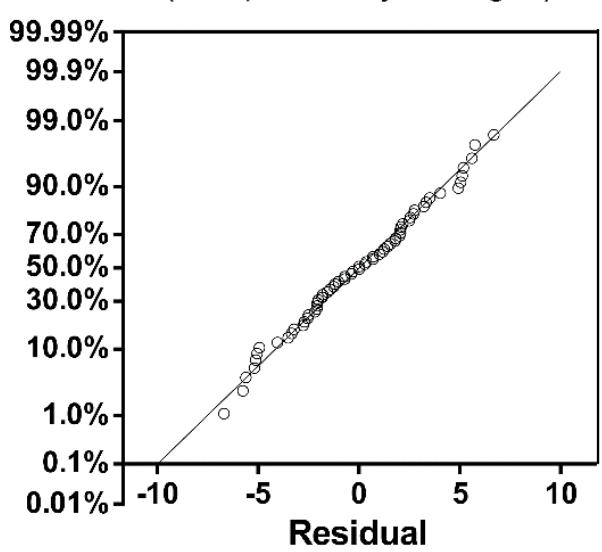

Pareto chart of standardized effects

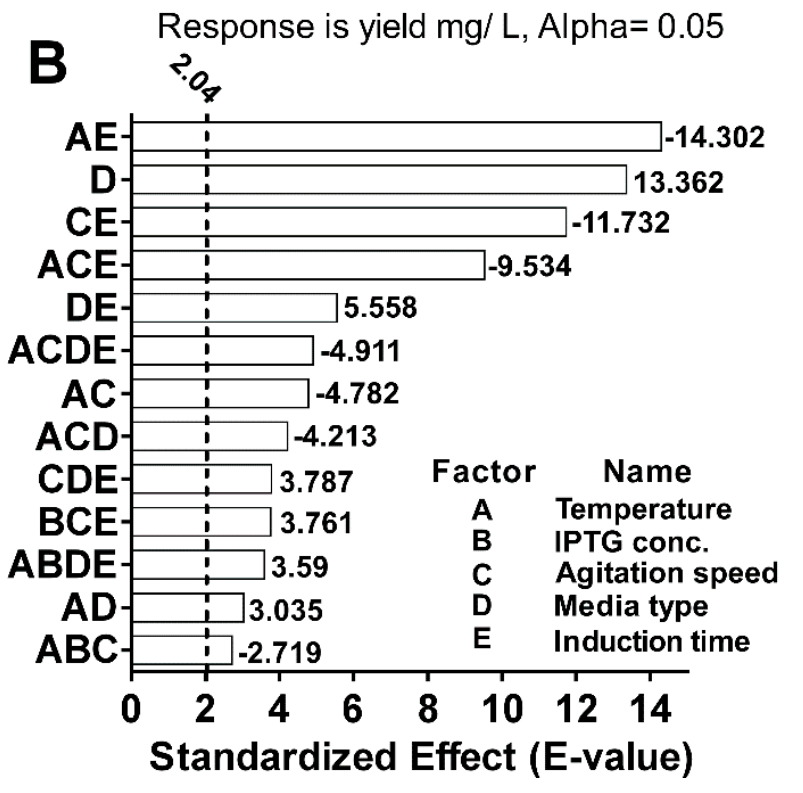

Figure 2. Normal probability plot of residuals and Pareto chart of standardized effects. (A). A normal probability plot of residuals. The straight line is a graphical representation of the mathematical regression equation and it represents the expected data, while the dots in the plots represents the actual observed data. (B). Pareto chart of standardized effects. Bars represent the significant interactions in the current model from the highest to the lowest regardless of the effect sign. The vertical dotted line represents the reference line and any factor that extends past this line is of significant effect at $\alpha=0.05$ (significance level). The magnitude of the E-value of the tested factor indicated its significance in affecting the response, while the positive or negative sign of the E-value was an indication of its positive or negative influence on the responses, respectively. In both charts, data was generated using Minitab 16 and plotted using GraphPad Prism (v6). 
The interactions between different factors and their effect on the response are presented in the interaction plots (Figure 3). There was a strong interaction between the temperature and three other factors (induction time, agitation speed, and media type) even though the significance of the interactions varied. Meanwhile, it has a minimal interaction with IPTG concentration, which was not significant. In addition, induction time shows a strong interaction with both agitation speed and media type. However, there was a minimal interaction between the IPTG concentration and every other factor as well as the relationship between the agitation speed and the media type.

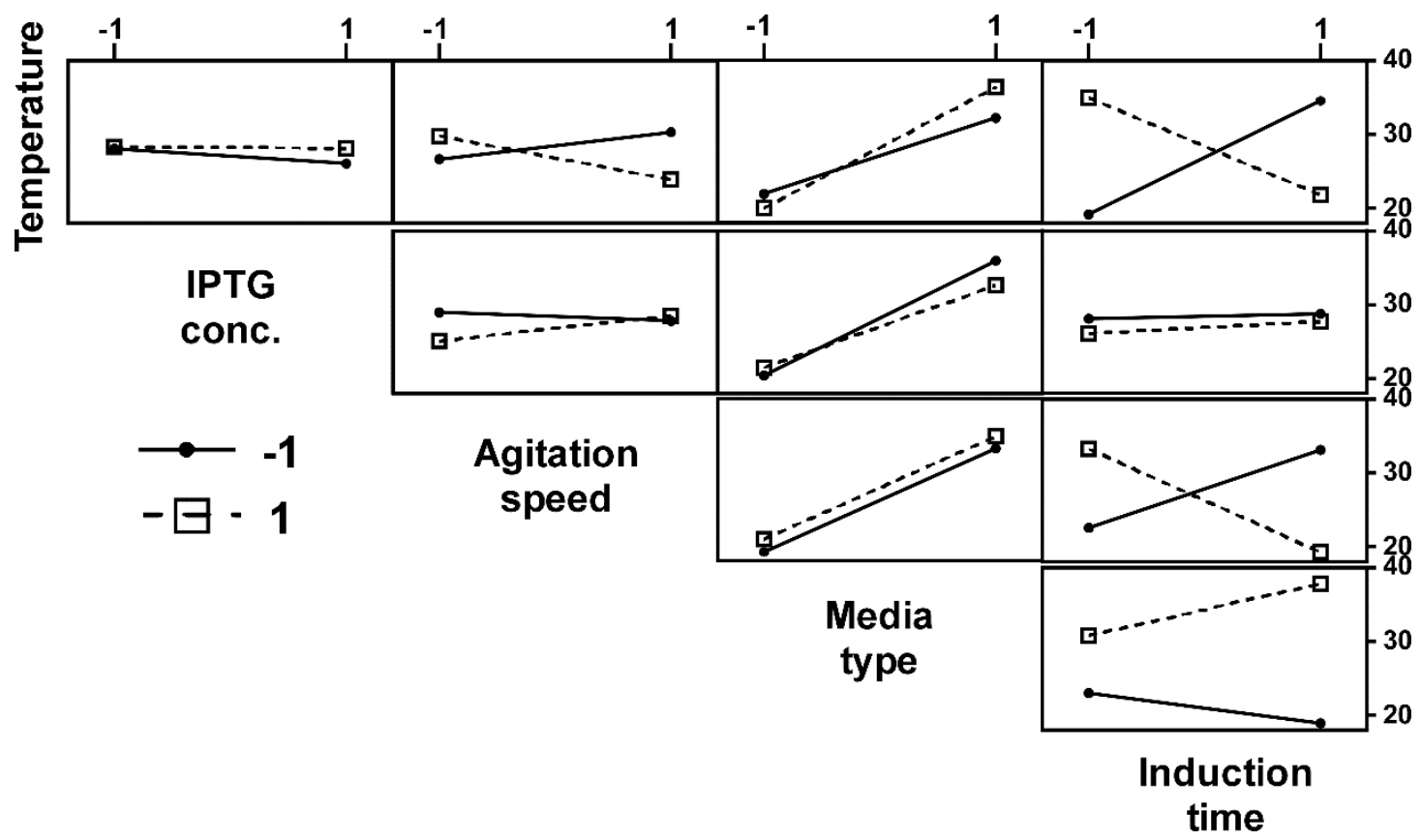

Figure 3. Interaction plot for the effects on rLST yield response. The interaction plot shows the possible interaction between the mean responses of factors under evaluation. The dotted line represents the high level (1) of the tested factor, while the solid line represents its low level (-1). The two levels (1 and -1$)$ of the tested factors are: temperatures of 37 and $16^{\circ} \mathrm{C}$, IPTG concentration of 1 and $0.25 \mathrm{mM}$, Agitation speed of 250 and $100 \mathrm{rpm}$, media type of TSB and NB, and induction time of 18 and $3 \mathrm{~h}$, respectively. Data was generated using Minitab 16 (Minitab, Inc., State College, Pennsylvania, PE, USA) and plotted using GraphPad Prism (v6) (GraphPad, California, CA, USA).

Taking all this into consideration, the optimum rLST yield was obtained in the 14th and 29th runs, both at rich media and high agitation speed levels, but with IPTG at a low level. High temperature level and low induction time were used in Run \#14 and low temperature level and high induction time were used in Run \#29.

\subsection{Laboratory-Scale Production Cost of rLST}

In order to evaluate the economic value of our applied system, we calculated the costs of rLST production using the conditions of Run \#14 (taking into account the prices of: culture media, Ni-NTA beads, IPTG, and the size exclusion chromatography columns for buffer exchange, being the major contributors to the rLST production in our settings, in addition to the labor costs). Producing $~ 2666$ units would cost about 7 EUR. Accordingly, the cost of one unit is $\sim 0.003$ EUR. Nevertheless, high scale production using our system will require further evaluation and more accurate calculations.

\subsection{The rLST Is Active in Lysing Staphylococcal Cells}

The rLST was tested against five staphylococcal strains in terms of reducing the $\mathrm{OD}_{600}$ of a cell suspension. As expected, rLST was totally non-effective against the immune species $S$. simulans strain 
(Figure 4A). The rLST showed the highest activity against the $S$. aureus strains especially strain Newman and was also effective against the MRSA strain (USA300). The reduction of the $\mathrm{OD}_{600}$ in both strains was statistically significant when compared to the immune cells of $S$. simulans. In addition, rLST showed significant activity against the commensal staphylococcal cells of S. epidermidis (Figure 4A). On the other hand, the activity of the rLST on the emerging in-dwelling device-related pathogen $S$. lugdunensis was very moderate and non-statistically significant from that on S. simulans (Figure 4A).
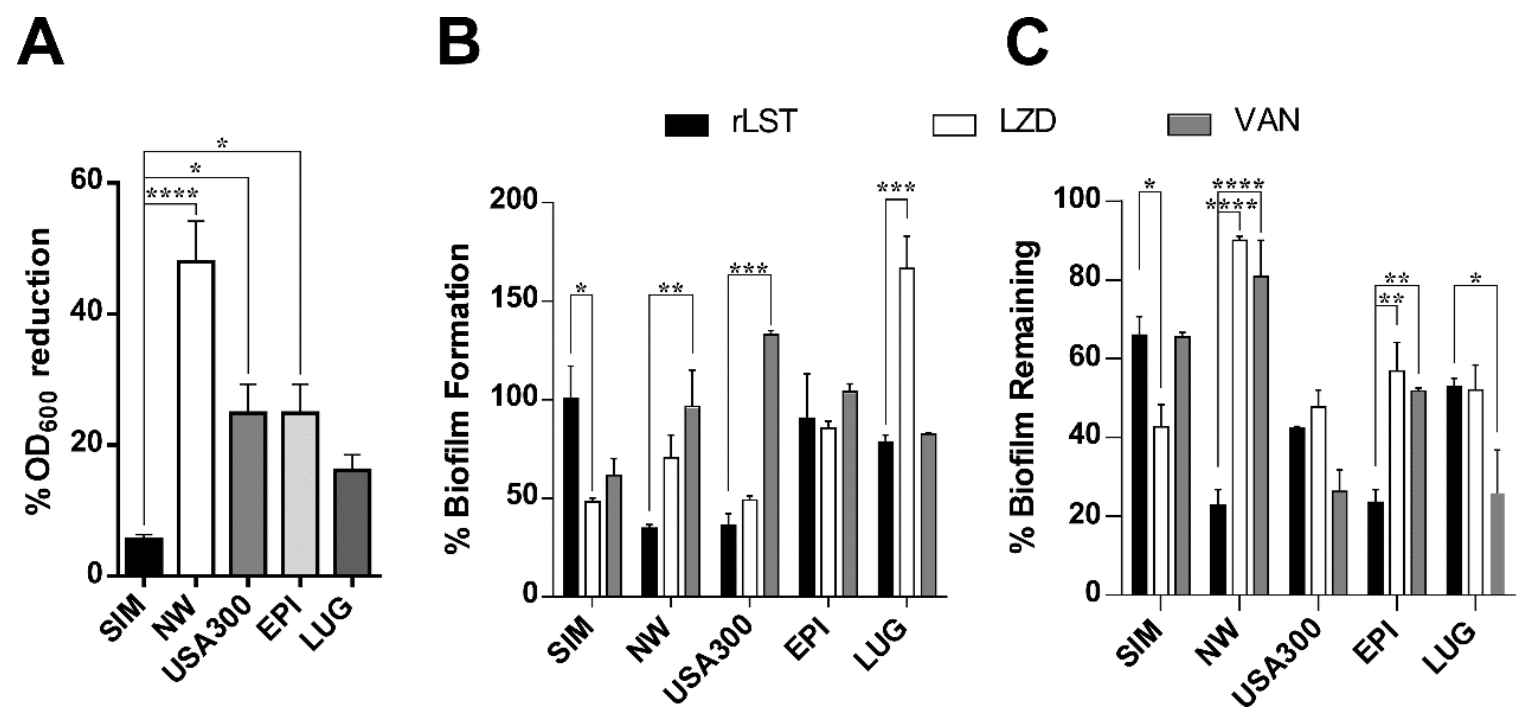

Figure 4. A. The rLST is active in-vitro against staphylococcal cells. (A). Bacteriolytic activity assay. Staphylococcal cells (S. simulans, SIM, S. aureus strain Newman, NW, S. aureus strain USA300, USA300, S. epidermidis, EPI, and S. S. lugdunensis, LUG) were suspended in $0.1 \mathrm{M}$ phosphate buffer ( $\mathrm{pH} 7.5$ ) to final $\mathrm{OD}_{600}$ of 0.25 , and then incubated with $50 \mu \mathrm{L}$ of $\mathrm{rLST}$ at $37^{\circ} \mathrm{C}$. Data presented as the percent reduction in $\mathrm{OD}_{600}$ after $30 \mathrm{~min}$. Bars represent the mean of four experiments and error bars are the standard error. Statistical analysis was done using a one-way ANOVA test followed by Dunnett's multiple comparisons test (a $p$ value of $\leq 0.05$ was considered significant). (B). Biofilm inhibition assay (Pre-exposure assay). Biofilm combating ability of rLST, black bars, linezolid (LZD), white bars, and vancomycin (VAN). Grey bars were tested using a crystal violet-based assay against the five staphylococcal cells mentioned above. Cells were grown in the absence and presence of the respective antimicrobial agent and, then, attached cells were stained with crystal violet. The fixed stain was then extracted with $99 \%$ ethanol and quantified spectrophotometrically at $595 \mathrm{~nm}$. Bars represent the increases or decreases in biofilm formation compared to the biofilm formation level in the absence of the antimicrobial agents for each strain. Data presented is the average of two independent experiments with each one done in triplicate and error bars represent the standard error. (C). Biofilm detachment assay (Post-exposure assay). Biofilms already formed by the five staphylococcal strains were exposed to 100X MIC of both LZD and VAN and $10 \mathrm{U}$ rLST for $24 \mathrm{~h}$ at $37^{\circ} \mathrm{C}$. The remaining biofilms were quantified as described above. Data presented is the average of two independent experiments with each one done in triplicate and error bars represent the standard error. In both B and C, statistical analysis was done using two-way ANOVA, which was followed by Tukey's multiple comparisons test with a significance level at $p \leq 0.05$. In all three charts, ${ }^{*}$ means $p \leq 0.05,{ }^{* *}$ means $p \leq 0.01,{ }^{* * *}$ means $p \leq 0.001$, and ${ }^{* * * *}$ means $p \leq 0.0001$. The charts were generated using GraphPad Prism (v6).

\subsection{The rLST Is Active in Inhibiting and Combating Staphylococcal Biofilms}

The clearest effect of rLST was shown in inhibiting the biofilm formation of the two S. aureus strains (Newman and USA300) when compared to vancomycin (Figure 4B). On the other hand, it did not show significant inhibition of the biofilm formation in either S. epidermidis nor S. lugdunensis. It was observed that S. lugdunensis in the presence of sub-MIC of linezolid showed enhanced biofilm formation (Figure 4B). 
Upon testing the activity of the three antimicrobial agents in the detachment of already formed biofilms, S. simulans was the most resistant species to rLST while S. aureus Newman and S. epidermidis were the most sensitive (Figure 4C). The rLST showed significant superior activity over both linezolid and vancomycin in the detachment of the biofilms formed by the S. aureus strain Newman and S. epidermidis. However, in the case of strain USA300, both linezolid and vancomycin were capable of detaching already formed biofilms to a degree very comparable to that of rLST (Figure 4C).

\subsection{The rLST Is Successfully Incorporated into a Nano-Emulgel and Released While Retaining Its Activity}

The prepared nano-emulgel was transparent with a yellowish color appearance. TEM examination showed spherical water globules with particle size-values ranging in the nano-size (Figure 5A). It was observed that rLST was released gradually over a prolonged time (Figure 5B) with the highest amount released after $8 \mathrm{~h}$. The released fractions were analyzed using the activity assay and rLST showed the highest activity in the fraction released after $5 \mathrm{~h}$. However, up to $8 \mathrm{~h}$, the activity remained relatively high (Figure 5B).
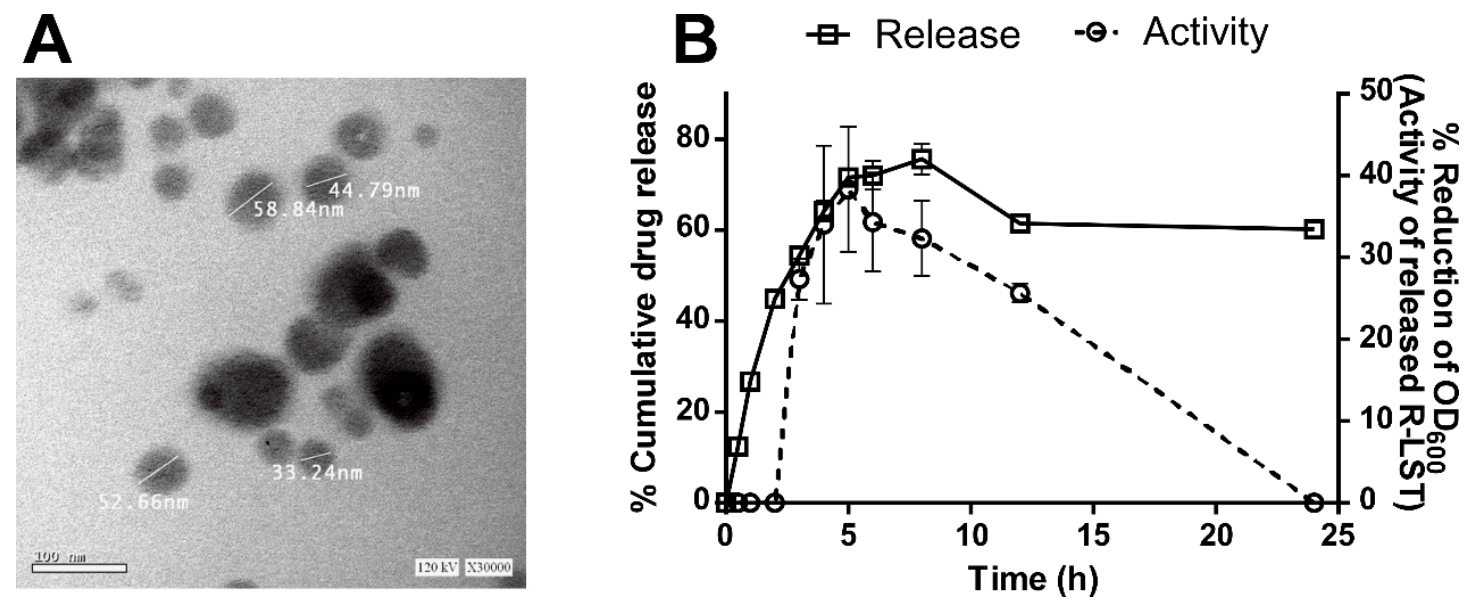

Figure 5. The recombinant lysostaphin (rLST) is successfully incorporated into a nano-emulgel and released while retaining its activity. (A) The formula particle is within the nano-range. High resolution-transmission electron microscopy image showing the globular structure of the prepared formula and displaying the size of the four representative nanoparticles. The photograph was obtained by an HR-TEM - JEOL2100 coupled with a Gatan axis-mount 2kx2k digital camera. (B). rLST is released from the formula in an active form. A chart showing the percentage of cumulative rLST release from the nano-emulgel formula (the left Y-axis and the solid line with open squares) over time in hours at the indicated time points (the $\mathrm{x}$-axis). The released rLST was assayed for its bacteriolytic activity as measured by the decrease in the $\mathrm{OD}_{600}$ of the staphylococcal cells (the right $\mathrm{Y}$-axis and the dotted line with open circles). The data presented is the average of two independent experiments each done in duplicate and the error bars represent the standard error. The charts were generated using GraphPad Prism (v6).

\subsection{The rLST Is Active in Curing S. aureus Skin Infection in Mice}

Mice in the control group showed lesions that are ulcerated and filled with pus (Figure 6A) and the lesion extended over a large area. A similar trend was seen with the group treated with the vehicle only. On the other hand, in the mice treated with rLST, the lesions healed to a great extent and the size was significantly less than that of the control group (Figure 6B). The bacterial counts of S. aureus cells recovered from the mice group treated with the rLST yielded average counts that were 2.747 logs lower than those yielded from the control group and 1.671 logs lower than that of the vehicle-treated group (Figure 6C). 


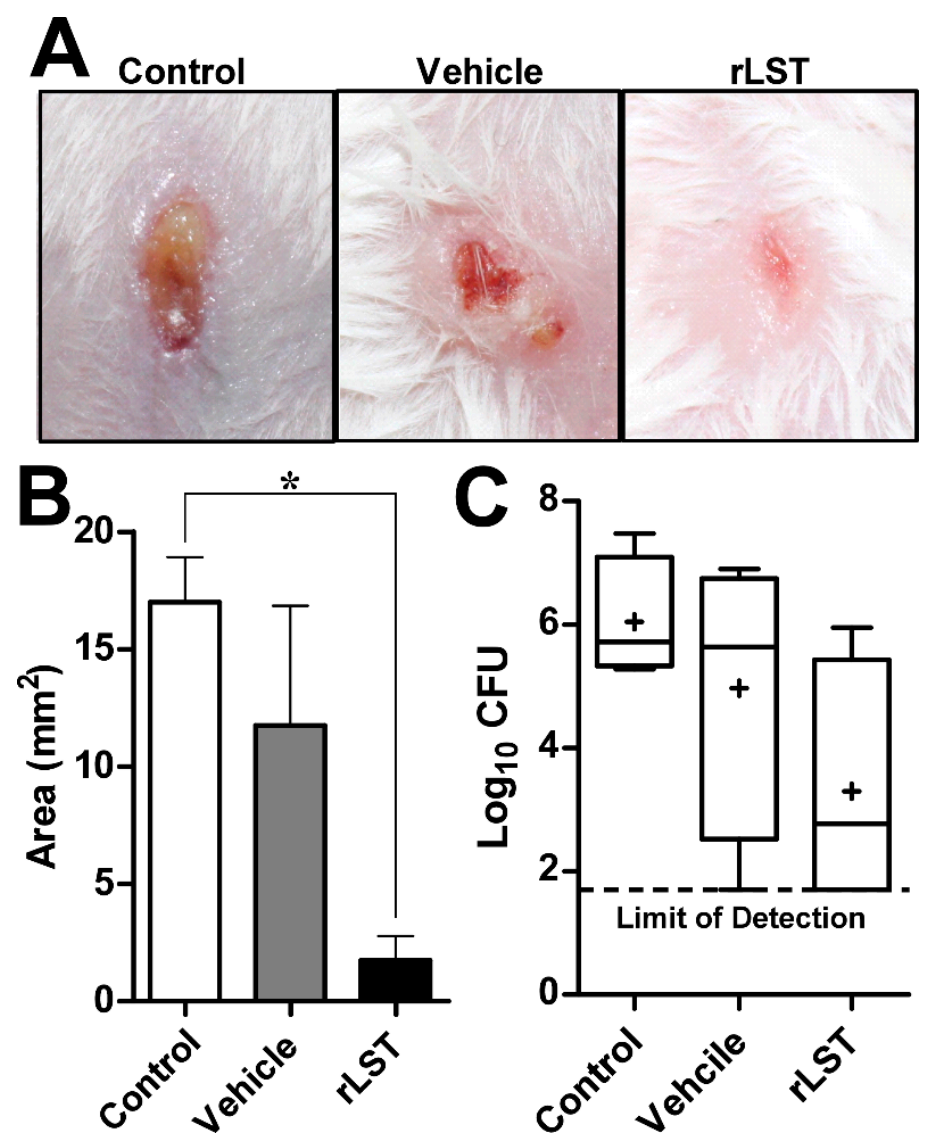

Figure 6. The rLST is active in-vivo curing $S$. aureus skin infection in mice. Mice were infected intradermally with approximately $3 \times 10^{7}$ CFUs of $S$. aureus USA300. Forty-eight hours after infection, an open wound/abscess was formed at the site of injection. Mice were then treated topically, three times daily for five days, with either rLST or vehicle alone (Vehicle) and no treatment at all (Control). (A). Photographs of representative mice showing the infected and treated skin areas in the three groups. (B). Bar chart showing the lesion surface area $\left(\mathrm{mm}^{2}\right)$ for the control group (white bar), vehicle group (grey bar), and R-LST (black bar). Statistical analysis was done using One-way ANOVA followed by Dunnett's multiple comparisons test ( $\mathrm{a} p^{*}$ value of $\leq 0.05$ was considered significant) and error bars represent the standard error. ${ }^{*}$ indicates that the $p$ value is $\leq 0.05$. (C). Box plot of the bacterial burden recovered from the lesions of the mice of the three groups. The whiskers span the difference between the minimum and maximum readings, the horizontal bar represents the median, and the (+) sign represents the mean of the $\log _{10} \mathrm{CFU}$. The horizontal dotted line represents the limit of detection of the experiment, which is 1.69897 ( $\log _{10}$ of $\left.50 \mathrm{CFU}\right)$. Statistical analysis was done using One-way ANOVA, which is followed by Dunnett's multiple comparisons test (a $p$ value of $\leq 0.05$ was considered significant) and error bars represent the standard error.

\section{Discussion}

In this study, we aimed to introduce a non-conventional antimicrobial agent to add to the antimicrobial arsenal needed to fight MDR-infections caused by S. aureus. Such an agent is urgently needed especially for countries with limited resources that cannot afford the costs of newly innovated antimicrobial agents. To this end, we optimized the production of recombinant lysostaphin expressed with the E. coli BL21 (DE3)/pET15b system to minimize both costs and time for production. Although this is not the first attempt to produce this biological macromolecule, we managed using factorial optimization to obtain a protein yield that surpassed comparable reported systems. For instance, previous studies used the E. coli BL21 (DE3) host combined with various expression systems including 
the pET28a yielded $22 \mathrm{mg} / \mathrm{L}$ [33], pET23b yielded $20 \mathrm{mg} / \mathrm{L}$ [34], pET15b yielded $11 \mathrm{mg} / \mathrm{L}$ [23], pET32a yielded $30 \mathrm{mg} / \mathrm{L}$ [35], and the current study yielded $50 \mathrm{mg} / \mathrm{L}$.

Factorial design is a widely applied precise experimental tool that successfully studies the main factors effect and interaction effect of factors on the response. In the present study, five-factor two-level full factorial optimization experiments identified conditions that yielded up to $50 \mathrm{mg} / \mathrm{L}$ purified protein using as low as $0.25 \mathrm{mM}$ IPTG. For the media type, the nutritious and buffered nature of TSB added an edge over the other two studied media and was the most significant factor in the main effects. Additionally, combining this with growth at $37^{\circ} \mathrm{C}$ and good aeration of the culture (high agitation speed) managed to minimize the induction time to $3 \mathrm{~h}$. It was also found that proper mixing via a high agitation rate is important for better induction.

For the purification purpose, we chose the Ni-NTA affinity chromatography since it allows simple one-step protein purification and does not require expertise or the equipment necessary for traditional protein purification schemes [35]. The relative high-cost of the nickel beads is overcome by the fact that they can be reused after being easily regenerated and recharged.

Overall, the production of pure active rLST took five hours while taking into consideration the induction time, sonication, purification, and preparatory steps. Cost-wise, the produced rLST costs $\sim 0.003$ EUR per unit of enzyme activity. This cost is very comparable to the cost reported by Szweda and co-workers [36]. However, the latter product was on a larger scale than that adopted in our current study. Accordingly, upscaling our production is expected to result in more savings per unit cost. Commercially, LST is available with around 137 EUR for 500 units, making the cost of one rLST unit 0.27 EUR. Due to the relatively high cost of this in-market product, commercial LST is not intended for clinical use.

Staphylococci are responsible for a significant proportion of the biofilm-based infections, which are challenging to healthcare facilities $[37,38]$. Biofilm combating ability of rLST using pre-exposure assay showed a superior biofilm inhibition effect on both the Newman strain and the MRSA strain USA300, (Figure 4B). As noted, sub-MIC concentrations of vancomycin and linezolid not only had a lower biofilm inhibition effect but also augmented the biofilm formation ability at certain instances such as in the case S. lugdunensis with linezolid, and USA300 when treated with vancomycin. The enhanced biofilm formation ability was previously explained as a response phenomenon to cell stress caused by cell wall-active antibiotics such as oxacillin, cephalothin, cephalexin, and vancomycin $[39,40]$ and the protein synthesis inhibitor linezolid [41]. On the other hand, LST acts on the sessile staphylococci, destabilizing the whole biofilm matrix, but those staphylococci have to be LST-sensitive [28], which explains the strain-dependent pattern noticeable with rLST assays on preformed biofilms. Accordingly, rLST managed to significantly interfere with the formation of new biofilms and the maintenance of already formed ones.

The greatest fear that accompanies the introduction of an antimicrobial agent to the therapeutic field is the rapid development of resistance. Strandén and co-workers have reported the emergence of resistance to LST [42]. Yet, there are no reported LST-resistant mutants from in-vivo studies employing high doses of LST [10]. The most common resistance mechanism known for LST is the development of mutations in either the $f e m A$ or the $f e m B$ genes, which results in mono-glycine or tri-glycine cross bridges in the cell wall [42]. These resistant mutants were less fit and less virulent. Hence, the LST resistance caveat was easily overcome by adjunct beta-lactam antibiotics therapy [43].

The use of LST to treat infections has been demonstrated before, using different routes of administration, and it showed encouraging results modeling this bacteriocin as a promising antibiotics-alternative and a supporting adjuvant as well $[44,45]$. One of the most common LST administration routes was intravenous for staphylococcal infections of various degrees [44-47]. This route would be sensible for systemic infections. Yet, for skin infections, drug delivery through the skin is preferred for offering drug targeting ability over a prolonged period of time, and practicality of patient's self-management of drug administration [48]. Moreover, one of the most appealing lysostaphin merits that makes it a suitable nominee for topical preparations above other formulas is 
its high specificity for staphylococcal species [49]. Therefore, it is unlikely to distress or negatively affect the normal microflora of the skin, which itself provides a protective barrier against colonizing or opportunistic pathogens [50].

Topical LST preparations previously reported included the use of petroleum-based creams [28], gel incorporated LST [50], and lysostaphin formulated in petroleum jelly [51]. These topical agents are normally very sticky with a lower spreading coefficient. Hence, they need to be applied with rubbing, which causes uneasiness of application and lowers patients' adherence to treatment. Additionally, they tend to exhibit low stability [13]. Whereas, NEs are resistant to creaming, flocculation, and sedimentation of high stability. Another formula was made of chitosan-o/w cream incorporated with lysostaphin [52] that was useful for resolving S. aureus nasal colonization. Nasal application required a thick-based formula to stand in the face of mucociliary clearance that greatly limited LST delivery in the nasal cavity. However, topical skin preparations do not face such a problem and require other characteristics to make it more convenient to the patients. Moreover, the chitosan-o/w cream preparation procedure used seven components with heating and cooling of the aqueous and lipid phase before LST addition, which makes it more time and cost-consuming if considered for scaling-up.

Lysostaphin, which is a heat-labile protein, was required to be incorporated in a formula with a low-energy technique. The phase inversion composition (PIC) method, which required no heating, fulfilled this aim, with the edge of having an easy preparatory method. We were able to extend the LST release while maintaining its activity up to $8 \mathrm{~h}$. This prolonged rLST release was expected since it was incorporated in the internal phase of the nano-emulgel. Therefore, the drug should be diffused from the internal phase to the external phase and then to the release media in order to be detected. To test the efficacy of the formula, the murine skin model was chosen since abscesses are the hallmarks of staphylococcal infections. Abscess lesions tend to harbor high titers of slowly multiplying bacteria, which makes antibiotic failure highly likely to happen [10]. The rLST managed to enhance the recovery of the mice from the infection, as indicated by the decrease of both the surface area of the formed lesions and the bacterial burden within the skin.

\section{Conclusions}

The current study models an expeditious rLST production protocol from a simple rapid enzyme expression directly to a topical formula of high commercial potential as an alternative antimicrobial agent for treating methicillin-resistant $S$. aureus skin infections. This work establishes a rLST production platform that aims to satisfy the needs of countries with limited resources in order to be able to combat the surge in the MDR S. aureus infections.

Supplementary Materials: The following are available online at http://www.mdpi.com/2218-273X/10/3/435/s1.

Author Contributions: Conceptualization, methodology, data curation: H.T.N.E.-D., N.M.E., M.A.E.-G., and A.S.A., A.A.M., and M.M.M.H. have contributed to the analysis and interpretation of the data. Writing-original draft preparation, H.T.N.E.-D., N.M.E., M.A.E.G., A.S.A., A.A.M., and M.M.M.H. All authors have read and agreed to the published version of the manuscript.

Funding: This work was mainly self-funded by the authors and in part through the Cairo University funding system for assistant lecturers.

Acknowledgments: The authors would like to thank Eric P. Skaar of Vanderbilt University Medical Center, USA for providing bacterial strains.

Conflicts of Interest: The authors declare no conflict of interest.

\section{References}

1. Boucher, H.W.; Talbot, G.H.; Bradley, J.S.; Edwards, J.E.; Gilbert, D.; Rice, L.B.; Scheld, M.; Spellberg, B.; Bartlett, J. Bad Bugs, No Drugs: No ESKAPE! An Update from the Infectious Diseases Society of America. Clin. Infect. Dis. 2009, 48, 1-12. [CrossRef] [PubMed]

2. Pendleton, J.N.; Gorman, S.P.; Gilmore, B.F. Clinical relevance of the ESKAPE pathogens. Expert Rev. Anti-Infect. Ther. 2013, 11, 297-308. [CrossRef] [PubMed] 
3. W.H.O. Prioritization of Pathogens to Guide Discovery, Research and Development of New Antibiotics for Drug-Resistant Bacterial Infections, Including Tuberculosis; World Health Organization: Geneva, Switzerland, 2017.

4. Savoia, D. Plant-derived antimicrobial compounds: alternatives to antibiotics. Future Microbiol. 2012, 7 , 979-990. [CrossRef] [PubMed]

5. Burrowes, B.; Harper, D.R.; Anderson, J.; McConville, M.; Enright, M.C. Bacteriophage therapy: potential uses in the control of antibiotic-resistant pathogens. Expert Rev. Anti-Infect. Ther. 2011, 9, 775-785. [CrossRef]

6. Kole, R.; Krainer, A.R.; Altman, S. RNA therapeutics: beyond RNA interference and antisense oligonucleotides. Nat. Rev. Drug Discov. 2012, 11, 125-140. [CrossRef]

7. El-Halfawy, O.M.; Czarny, T.L.; Flannagan, R.S.; Day, J.; Bozelli, J.C.J.; Kuiack, R.C.; Salim, A.; Eckert, P.; Epand, R.M.; McGavin, M.J.; et al. Discovery of an antivirulence compound that reverses beta-lactam resistance in MRSA. Nat. Chem. Biol. 2019, 16, 143-149. [CrossRef]

8. Li, Y.; Xiang, Q.; Zhang, Q.; Huang, Y.; Su, Z. Overview on the recent study of antimicrobial peptides: Origins, functions, relative mechanisms and application. Peptides 2012, 37, 207-215. [CrossRef]

9. Bannerman, T.L. Staphylococcus, Micrococcus, and other catalase-positive cocci that grow aerobically. Man. Clin. Microbiol. 2003, 8, 384-404.

10. do Carmo de Freire Bastos, M.; Coutinho, B.G.; Coelho, M.L.V. Lysostaphin: a staphylococcal bacteriolysin with potential clinical applications. Pharmaceuticals 2010, 3, 1139-1161. [CrossRef]

11. Koroleva, M.Y.; Yurtov, E.V. Nanoemulsions: the properties, methods of preparation and promising applications. Russ. Chem. Rev. 2012, 81, 21. [CrossRef]

12. Azmi, N.A.N.; Elgharbawy, A.A.M.; Motlagh, S.R.; Samsudin, N.; Salleh, H.M. Nanoemulsions: Factory for Food, Pharmaceutical and Cosmetics. Processes 2019, 7, 617. [CrossRef]

13. Ojha, A.; Ojha, M.; Madhav, N.V.S. Recent Advancement in Emulgel: A Novel Approach for Topical Drug Delivery. Int. J. Adv. Pharm. 2017, 6, 1-21.

14. Patel, Z.S.; Shah, R.; Shah, D.N. Nanoemulsion: An innovative approach for topical delivery. Pharma Sci. Monit. 2016, 7, 21-36.

15. Salome Amarachi, C.; Kenechukwu, F.; Attama, A. Nanoemulsions - Advances in formulation, characterization and applications in drug delivery. In Application of Nanotechnology in Drug Delivery; Sezer, A.D., Ed.; IntechOpen: London, UK, 2014; pp. 76-126.

16. Lorenz, L.L.; Duthie, E.S. Staphylococcal Coagulase: Mode of Action and Antigenicity. Microbiology 1952, 6, 95-107.

17. Diep, B.A.; Gill, S.R.; Chang, R.F.; Phan, T.H.; Chen, J.H.; Davidson, M.G.; Lin, F.; Lin, J.; Carleton, H.A.; Mongodin, E.F.; et al. Complete genome sequence of USA300, an epidemic clone of community-acquired meticillin-resistant Staphylococcus aureus. Lancet 2006, 367, 731-739. [CrossRef]

18. Daniel, A.; Euler, C.; Collin, M.; Chahales, P.; Gorelick, K.J.; Fischetti, V.A. Synergism between a novel chimeric lysin and oxacillin protects against infection by methicillin-resistant Staphylococcus aureus. Antimicrob. Agents Chemother. 2010, 54, 1603-1612. [CrossRef] [PubMed]

19. Heilbronner, S.; Holden, M.T.G.; van Tonder, A.; Geoghegan, J.A.; Foster, T.J.; Parkhill, J.; Bentley, S.D. Genome sequence of Staphylococcus lugdunensis N920143 allows identification of putative colonization and virulence factors. FEMS Microbiol. Lett. 2011, 322, 60-67. [CrossRef]

20. Studier, F.W.; Moffatt, B.A. Use of bacteriophage T7 RNA polymerase to direct selective high-level expression of cloned genes. J. Mol. Biol. 1986, 189, 113-130. [CrossRef]

21. Recsei, P.A.; Gruss, A.D.; Novick, R.P. Cloning, sequence, and expression of the lysostaphin gene from Staphylococcus simulans. Proc. Natl. Acad. Sci. USA 1987, 84, 1127-1131. [CrossRef]

22. O'Connell, D.P.; Nanavaty, T.; McDevitt, D.; Gurusiddappa, S.; Höök, M.; Foster, T.J. The Fibrinogen-binding MSCRAMM (Clumping Factor) of Staphylococcus aureus has a $\mathrm{Ca}^{2+}$-dependent inhibitory site. J. Biol. Chem. 1998, 273, 6821-6829. [CrossRef]

23. Szweda, P.; Kotłowski, R.; Kur, J. New effective sources of the Staphylococcus simulans lysostaphin. J. Biotechnol. 2005, 117, 203-213. [CrossRef] [PubMed]

24. CLSI. M100 Performance Standards for Antimicrobial Susceptibility Testing an Informational Supplement for Global Application Developed through the Clinical and Laboratory Standards Institute Consensus Process; CLSI: Wayne, PA, USA, 2016; ISBN 1562388045.

25. O'Toole, G.A. Microtiter dish biofilm formation assay. J. Vis. Exp. 2011, 47, 2437. [CrossRef] [PubMed] 
26. He, X.; Yuan, F.; Lu, F.; Yin, Y.; Cao, J. Vancomycin-induced biofilm formation by methicillin-resistant Staphylococcus aureus is associated with the secretion of membrane vesicles. Microb. Pathog. 2017, 110, 225-231. [CrossRef] [PubMed]

27. Geoghegan, J.A.; Corrigan, R.M.; Gruszka, D.T.; Speziale, P.; O'Gara, J.P.; Potts, J.R.; Foster, T.J. Role of surface protein SasG in biofilm formation by Staphylococcus aureus. J. Bacteriol. 2010, 192, 5663-5673. [CrossRef]

28. Wu, J.A.; Kusuma, C.; Mond, J.J.; Kokai-Kun, J.F. Lysostaphin disrupts Staphylococcus aureus and Staphylococcus epidermidis biofilms on artificial surfaces. Antimicrob. Agents Chemother. 2003, 47, 3407-3414. [CrossRef]

29. Forgiarini, A.; Esquena, J.; González, C.; Solans, C. Formation of nano-emulsions by low-energy emulsification methods at constant temperature. Langmuir 2001, 17, 2076-2083. [CrossRef]

30. Mohamed, M.F.; Hamed, M.I.; Panitch, A.; Seleem, M.N. Targeting methicillin-resistant Staphylococcus aureus with short salt-resistant synthetic peptides. Antimicrob. Agents Chemother. 2014, 58, 4113-4122. [CrossRef]

31. Mohamed, M.F.; Abdelkhalek, A.; Seleem, M.N. Evaluation of short synthetic antimicrobial peptides for treatment of drug-resistant and intracellular Staphylococcus aureus. Sci. Rep. 2016, 6, 29707. [CrossRef]

32. Gasteiger, E.; Hoogland, C.; Gattiker, A.; Wilkins, M.R.; Appel, R.D.; Bairoch, A. Protein identification and analysis tools on the ExPASy server. In The Proteomics Protocols Handbook; Springer: New York, NA, USA, 2005; pp. 571-607.

33. Sharma, R.; Sharma, P.R.; Choudhary, M.L.; Pande, A.; Khatri, G.S. Cytoplasmic expression of mature glycylglycine endopeptidase lysostaphin with an amino terminal hexa-histidine in a soluble and catalytically active form in Escherichia coli. Protein Expr Purif. 2006, 45, 206-215. [CrossRef]

34. Zhang, B.; Shangguan, T.; Ma, H.; Huang, X.; Zhang, Y. Lysis of mastitis pathogens isolated from dairy cow milk samples by purified recombinant lysostaphin. Afr. J. Biotechnol. 2012, 11, 4649-4659.

35. Farhangnia, L.; Ghaznavi-Rad, E.; Mollaee, N.; Abtahi, H. Cloning, expression, and purification of recombinant lysostaphin from staphylococcus simulans. Jundishapur J. Microb. 2014, 7, e10009. [CrossRef] [PubMed]

36. Szweda, P.; Gorczyca, G.; Filipkowski, P.; Zalewska, M.; Milewski, S. Efficient production of Staphylococcus simulans lysostaphin in a benchtop bioreactor by recombinant Escherichia coli. Prep. Biochem. Biotech. 2014, 44, 370-381. [CrossRef] [PubMed]

37. Kiedrowski, M.R.; Horswill, A.R. New approaches for treating staphylococcal biofilm infections. Ann. N. Y. Acad. Sci. 2011, 1241, 104-121. [CrossRef]

38. Rasmussen, T.B.; Givskov, M. Quorum-sensing inhibitors as anti-pathogenic drugs. Int.J. Med. Microbiol. 2006, 296, 149-161. [CrossRef] [PubMed]

39. Kaplan, J.B. Antibiotic-induced biofilm formation. Int. J. Artif. Organs. 2011, 34, 737-751. [CrossRef] [PubMed]

40. Mirani, Z.A.; Jamil, N. Effect of sub-lethal doses of vancomycin and oxacillin on biofilm formation by vancomycin intermediate resistant Staphylococcus aureus. J. Basic Microbiol. 2011, 51, 191-195. [CrossRef]

41. Frank, K.L.; Reichert, E.J.; Piper, K.E.; Patel, R. In vitro effects of antimicrobial agents on planktonic and biofilm forms of Staphylococcus lugdunensis clinical isolates. Antimicrob. Agents Chemother. 2007, 51, 888-895. [CrossRef]

42. Stranden, A.M.; Ehlert, K.; Labischinski, H.; Berger-Bächi, B. Cell wall monoglycine cross-bridges and methicillin hypersusceptibility in a fem $\mathrm{AB}$ null mutant of methicillin-resistant Staphylococcus aureus. J. Bacteriol. 1997, 179, 9-16. [CrossRef]

43. Kusuma, C.; Jadanova, A.; Chanturiya, T.; Kokai-Kun, J.F. Lysostaphin-resistant variants of Staphylococcus aureus demonstrate reduced fitness in vitro and in vivo. Antimicrob. agents Chemother. 2007, 51, 475-482. [CrossRef]

44. Harrison, E.; Cropp, C. Therapeutic activity of lysostaphin in experimental staphylococcal infections. Can. J. Microbiol. 2011, 13, 93-97. [CrossRef]

45. Climo, M.W.; Patron, R.L.; Goldstein, B.P.; Archer, G.L. Lysostaphin treatment of experimental methicillinresistant Staphylococcus aureus aortic valve endocarditis. Antimicrob. Agents Chemother. 1998, 42, 1355-1360. [CrossRef] [PubMed]

46. Kiri, N.; Archer, G.; Climo, M.W. Combinations of lysostaphin with beta-lactams are synergistic against oxacillin-resistant Staphylococcus epidermidis. Antimicrob. Agents Chemother. 2002, 46, 2017-2020. [CrossRef] [PubMed] 
47. Placencia, F.X.; Kong, L.; Weisman, L.E. Treatment of methicillin-resistant Staphylococcus aureus in neonatal mice: lysostaphin versus vancomycin. Pediatr. Res. 2009, 65, 420-424. [CrossRef] [PubMed]

48. Devarajan, V.; Ravichandran, V. Nanoemulsions: As modified drug delivery tool advantages of nanoemulsion. Int. J. Compr. Pharm. 2011, 2,1-6.

49. Schindler, C.; Schuhardt, V. Lysostaphin: A new bacteriolytic agent for the staphylococcus. Proc. Natl. Acad. Sci. USA 1964, 51, 414-421. [CrossRef] [PubMed]

50. Desbois, A.P.; Lang, S.; Gemmell, C.G.; Coote, P.J. Surface disinfection properties of the combination of an antimicrobial peptide, ranalexin, with an endopeptidase, lysostaphin, against methicillin-resistant Staphylococcus aureus (MRSA). J. Appl. Microbiol. 2010, 108, 723-730. [CrossRef]

51. Mohamed, M.F.; Seleem, M.N. Efficacy of short novel antimicrobial and anti-inflammatory peptides in a mouse model of methicillin-resistant Staphylococcus aureus (MRSA) skin infection. Drug Des. Devel. Ther. 2014, 8, 1979-1983. [CrossRef]

52. Cui, F.; Li, G.; Huang, J.; Zhang, J.; Lu, M.; Lu, W.; Huang, Q. Extension of nasal anti-Staphylococcus aureus efficacy of lysostaphin by its incorporation into a chitosan-o/w cream. Drug Deliv. 2010, 17, 617-623. [CrossRef]

(C) 2020 by the authors. Licensee MDPI, Basel, Switzerland. This article is an open access article distributed under the terms and conditions of the Creative Commons Attribution (CC BY) license (http://creativecommons.org/licenses/by/4.0/). 